\title{
Thermal switching of the scattering coefficients of terahertz surface plasmon polaritons impinging on a finite array of subwavelength grooves on semiconductor surfaces
}

\section{Citation for published version (APA):}

Sánchez-Gil, J. A., \& Gómez Rivas, J. (2006). Thermal switching of the scattering coefficients of terahertz surface plasmon polaritons impinging on a finite array of subwavelength grooves on semiconductor surfaces. Physical Review B, 73(15), 205410-1/8. [205410]. https://doi.org/10.1103/PhysRevB.73.205410

DOI:

10.1103/PhysRevB.73.205410

Document status and date:

Published: 01/01/2006

\section{Document Version:}

Publisher's PDF, also known as Version of Record (includes final page, issue and volume numbers)

\section{Please check the document version of this publication:}

- A submitted manuscript is the version of the article upon submission and before peer-review. There can be important differences between the submitted version and the official published version of record. People interested in the research are advised to contact the author for the final version of the publication, or visit the DOI to the publisher's website.

- The final author version and the galley proof are versions of the publication after peer review.

- The final published version features the final layout of the paper including the volume, issue and page numbers.

Link to publication

\footnotetext{
General rights

- You may freely distribute the URL identifying the publication in the public portal. follow below link for the End User Agreement:

www.tue.nl/taverne

\section{Take down policy}

If you believe that this document breaches copyright please contact us at:

openaccess@tue.nl

providing details and we will investigate your claim.
}

Copyright and moral rights for the publications made accessible in the public portal are retained by the authors and/or other copyright owners and it is a condition of accessing publications that users recognise and abide by the legal requirements associated with these rights.

- Users may download and print one copy of any publication from the public portal for the purpose of private study or research.

- You may not further distribute the material or use it for any profit-making activity or commercial gain

If the publication is distributed under the terms of Article $25 \mathrm{fa}$ of the Dutch Copyright Act, indicated by the "Taverne" license above, please 


\title{
Thermal switching of the scattering coefficients of terahertz surface plasmon polaritons impinging on a finite array of subwavelength grooves on semiconductor surfaces
}

\author{
José A. Sánchez-Gil* \\ Instituto de Estructura de la Materia, Consejo Superior de Investigaciones Científicas, Serrano 121, 28006 Madrid, Spain \\ Jaime Gómez Rivas \\ FOM Institute for Atomic and Molecular Physics AMOLF, clo Philips Research Laboratories, High-Tech Campus 4 , \\ 5656 AE Eindhoven, The Netherlands
}

(Received 23 December 2005; published 15 May 2006)

\begin{abstract}
We present a theoretical framework that allows us to investigate the scattering of terahertz surface plasmon polaritons (SPP's) by arrays of subwavelength grooves and ridges on semiconductors. The formulation is based on the reduced Rayleigh equation resulting upon imposing an impedance boundary condition. Guided by approximate estimations of the broadening with temperature of the first gap in the SPP dispersion relation in the case of indium antimonide samples with rectangular grooves, numerical calculations are carried out to determine the spectral dependence of all the SPP scattering channels (reflection, transmission, and radiation) in the immediate vicinity of that gap. The thermally induced switching of the SPP reflection and transmission nearby the lower SPP band edge is investigated as a function of groove parameters (size and number); near-field intensity maps are also presented. We thus shed light on the SPP scattering and switching physical mechanisms, thereby providing the most suitable experimental configurations.
\end{abstract}

DOI: 10.1103/PhysRevB.73.205410 PACS number(s): 73.20.Mf, 78.68. $+\mathrm{m}, 65.40 .-\mathrm{b}$, 41.20.Jb

\section{INTRODUCTION}

Surface plasmon polaritons (SPP's) have been the subject of intense research in recent years. These are electromagnetic waves bound to the interface separating two media with the real part of the dielectric permittivities of opposite sign, one of them thus supporting plasma oscillations. ${ }^{1}$ Their unique properties confer them under certain conditions a twodimensional character and make them specially suitable for applications in subwavelength optics. ${ }^{2,3}$ Most of the effort has been concentrated on investigating SPP's in the visible (and near-IR) domain on metal surfaces, with the challenge of manipulating SPP's at the nanoscale. ${ }^{4}$ However, not only metals support electron plasma oscillations; semiconductors can also support SPP's, free carriers being excited either thermally or by ( $n$ or $p$ ) doping, with a resulting plasma frequency typically in the terahertz $(\mathrm{THz})$ domain. Subwavelength manipulation of SPP's at low frequencies is feasible, as confirmed by very recent experiments for groove arrays on doped-Si (Ref. 5) and indium antimonide. ${ }^{6}$ Terahertz SPP's considerably extend the electromagnetic spectrum and incorporate semiconductors as host materials for SPP optics.

Among the rich phenomenology explored for SPP's propagating on interfaces with subwavelength structures, particularly abundant for visible SPP's on nanostructured metals (cf. Ref. 4 and references therein), SPP scattering by periodic structures gives rise to a variety of phenomena under active research: full SPP band gaps, ${ }^{7-12}$ SPP reflection and interferometry, ${ }^{5,10,13-17}$ light-SPP coupling, ${ }^{10,14}$ SPP waveguiding, ${ }^{18}$ and dynamic effects of pulsed-SPP scattering. ${ }^{5,19}$ In this regard, it should be mentioned that all of the above-mentioned works deal with passive SPP scattering. Active plasmon optics-namely, the possibility of actively manipulating SPP's - undoubtedly means a big step forward in surface plasmon photonics; it can be particularly relevant in connection with SPP scattering and switching, as suggested by very recent experimental works with visible SPP' ${ }^{20,21}$ and THz-SPP's, ${ }^{6}$ both thermally controlled, and by the theoretical design based on modifying the SPP propagation medium. ${ }^{22}$

In this paper, we investigate theoretically the scattering of SPP by finite arrays of one-dimensional subwavelength grooves on thermally controlled InSb surfaces, rigorously accounting for all the scattering channels: SPP reflection and transmission and radiative leakage. The theoretical formulation employed is presented in Sec. II. An analytical formula to estimate the position of the first SPP gap is shown in Sec. III, thereby predicting the appropriate spectral region to exploit thermally induced gap shifts. Section IV contains rigorous numerical calculations for the SPP scattering efficiencies, along with near- and far-field scattered intensities, for finite arrays of grooves with different parameters (size and number), in all cases for a frequency range covering the first SPP band gap of the infinite array, with the aim of determining the optimum configurations to achieve high SPP switching efficiencies and the physical mechanism underlying the process.

\section{SPP SCATTERING GEOMETRY AND FORMULATION}

The system we consider is depicted in Fig. 1. A SPP of frequency $\omega$, whose plane of incidence is the $x z$ plane, propagates $^{1}$ along an interface separating vacuum $[z>f(x)]$ from a semiconductor $[z<f(x)]$ characterized by a Drudetype dielectric function

$$
\epsilon(\omega, T)=\epsilon_{\infty}-\frac{\omega_{p}^{2}(T)}{\omega[\omega+1 \Gamma(T)]},
$$

where $\epsilon_{\infty}$ is the high-frequency permittivity and $\omega_{p}(T)$ and $\Gamma(T)$ are, respectively, the plasma frequency and collision 


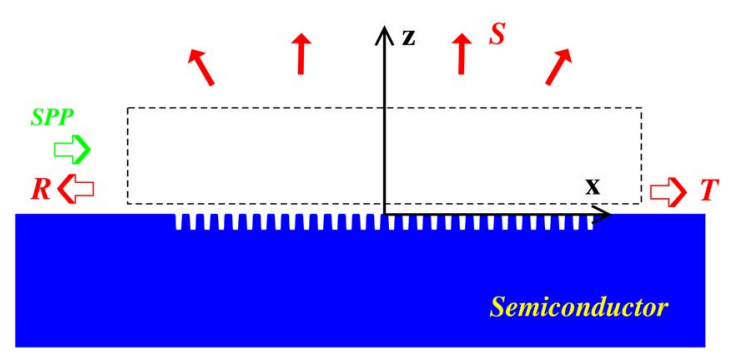

FIG. 1. (Color online) Illustration of the SPP scattering geometry. The array of grooves (parallel to the $y$ axis) is symmetrically located about the origin $x=0$. The incident SPP propagating on the semiconductor surface $(z=0)$ along the $x$ direction impinges from the negative $x$ axis on the groove array, being partially reflected (towards the negative $x$ axis), transmitted (towards the positive $x$ axis), and coupled to radiation into the vacuum region $z>0$. The dashed rectangle depicts the near-field area covered by the images in Figs. 4 and 6.

rate, which depend on temperature. Moderate changes of temperature modify $\epsilon(\omega)$ and, as we will see, the scattering characteristics of SPP's. The SPP impinges from the negative $x$ axes on a finite array of $N$ identical, parallel, equally spaced rectangular grooves of width $2 A$ and depth $h$, defined by the profile function $f(x)=\Sigma_{1}^{N} g[x-(n-1) d-N d / 2]$, where $g(x)=-h[\Theta(x+A)-\Theta(x-A)][\Theta(x)$ is the Heaviside step function]. Taking into account this scattering geometry and the fact that SPP's are $p$-polarized (transverse magnetic) waves, we deal only with the single nonzero component of the magnetic field. In the vacuum region, we write it as the sum of the incident SPP and a scattered field given as a Rayleigh (plane-wave) expansion:

$$
\begin{aligned}
H_{y}^{>}(x, z)= & \exp \left[i k(\omega) x-\beta_{0}(\omega) z\right]+\int_{-\infty}^{\infty} \frac{d q}{2 \pi} \mathcal{R}(q, \omega) \\
& \times \exp \left[i q x+i \alpha_{0}(q, \omega) z\right],
\end{aligned}
$$

with SPP wave vector

$$
\begin{aligned}
& k(\omega) \equiv \frac{\omega}{c}\left[\frac{\epsilon(\omega, T)}{\epsilon(\omega, T)+1}\right]^{1 / 2}, \\
& \beta_{0}(\omega) \equiv\left[k^{2}(\omega)-(\omega / c)^{2}\right]^{1 / 2} .
\end{aligned}
$$

$\mathcal{R}(q, \omega)$ is the scattering amplitude, and the perpendicular component of the scattered wave vector is given by

$$
\begin{aligned}
\alpha_{0}(q, \omega) & =\left(\frac{\omega^{2}}{c^{2}}-q^{2}\right)^{1 / 2} \quad\left(|q| \leq \frac{\omega}{c}\right) \\
& =i\left(q^{2}-\frac{\omega^{2}}{c^{2}}\right)^{1 / 2} \quad\left(|q|>\frac{\omega}{c}\right) .
\end{aligned}
$$

Note that the explicit temperature dependence has been omitted in Eqs. (2) and (3), which implicitly follows from that of the dielectric function. This is done throughout the paper for the sake of clarity, unless necessary to remark thermal properties.

We now impose the following local impedance boundary condition $^{23}$ on the planar interface $(z=0)$ :

$$
\left.\frac{\partial}{\partial z} H_{y}^{>}(x, z)\right|_{z=0}=-\left.\frac{\omega}{c} \frac{1+s(x)}{[-\epsilon(\omega, T)]^{1 / 2}} H_{y}^{>}(x, z)\right|_{z=0},
$$

where the superscript $>$ indicates the vacuum region. The above boundary condition maps the finite array of grooves onto a surface impedance contribution on a flat surface through $s(x)$ (to be discussed below). As a result, a reduced Rayleigh equation is obtained for the scattering amplitude $\mathcal{R}(q, \omega)$ (Ref. 24):

$$
\begin{aligned}
\mathcal{R}(q, \omega)= & G_{0}(q, \omega) V(q \mid k(\omega)) \\
& +G_{0}(q, \omega) \int_{-\infty}^{\infty} \frac{d p}{2 \pi} V(q \mid p) \mathcal{R}(p, \omega),
\end{aligned}
$$

where

$$
G_{0}(q, \omega) \equiv \frac{i \epsilon(\omega)}{\epsilon(\omega) \alpha_{0}(q, \omega)+i(\omega / c)[-\epsilon(\omega)]^{1 / 2}}
$$

is the Green's function of the SPP on the unperturbed surface $[s(x)=0]$ and

$$
V(q \mid p) \equiv \beta_{0}(\omega) \hat{s}(q-p)
$$

the scattering potential related to the Fourier transform of the surface impedance

$$
\hat{s}(Q)=\int_{-\infty}^{\infty} d x e^{-i Q x} s(x) .
$$

We proceed by reformulating Eq. (6) exactly as in Ref. 24. The resulting formulation allows us to obtain the SPP reflection and transmission coefficients $[R(\omega)$ and $\mathcal{T}(\omega)$, respectively] and the total power scattered into vacuum,

$$
S(\omega)=\int_{-\pi / 2}^{\pi / 2} d \theta_{s} \frac{\partial \mathcal{R}}{\partial \theta_{s}},
$$

properly normalized to the SPP incident power, where the differential reflection coefficient (DRC) is

$$
\frac{\partial \mathcal{R}}{\partial \theta_{s}}=\frac{1}{2 \pi} \frac{\beta_{0}(\omega)}{2 k(\omega)} \alpha_{0}^{2}\left(q=\frac{\omega}{c} \sin \theta_{s}\right)\left|\mathcal{R}\left(q=\frac{\omega}{c} \sin \theta_{s}\right)\right|^{2},
$$

which yields the fraction of the incident SPP energy scattered into an angular region of width $d \theta_{s}$ about the scattering direction $\theta_{s}$. Note that, in the absence of absorptive losses, $R(\omega)+\mathcal{T}(\omega)+S(\omega)=1$.

The surface impedance $s(x)$ has been shown to be connected to the actual surface profile $f(x)$ in the following manner ${ }^{23}$ :

$$
s(x)=-\frac{1-\epsilon(\omega, T)}{\delta(\omega, T) \epsilon(\omega, T)}\left[1-\delta^{2}(\omega, T)\right]^{1 / 2} f(x)=s_{0}(\omega, T) f(x),
$$

valid for small optical skin depth $\delta(\omega, T)=(c /$ $\omega)[-\epsilon(\omega, T)]^{1 / 2}$ and surface slopes. It has been successfully exploited to investigate a variety of SPP scattering phenomena on single grooves and ridges. ${ }^{24-26}$ 


\section{THERMAL DEPENDENCE OF THE BAND EDGE}

We aim to investigate the thermal control of SPP scattering by finite arrays of grooves. Before doing so, it is convenient to explore the thermal dependence of the SPP band gap in the infinite array configuration. For that purpose, we make use of Eq. (6) without incident field (first term of the righthand side) - namely, the homogeneous integral equation for $\mathcal{R}$-and exploit the periodic boundary conditions. Rather than solving the resulting truncated matrix equation for the corresponding terms of the Fourier series expansion of $\mathcal{R}$, we restrict ourselves to the first Bragg scattering of the SPP, which suffices to characterize the first SPP band gap. ${ }^{12,27}$ Thus we obtain the following analytical expression of the band edges for the first gap:

$$
\frac{\omega_{ \pm}(T)}{c} \approx \frac{G}{2}\left\{1-\frac{1}{\epsilon(T)}\left[1+s_{0}(T) f_{0} \mp\left|s_{0}(T) f_{1}\right|\right]^{2}\right\}^{-1 / 2},
$$

$G=2 \pi / d$ being the reciprocal lattice vector. Note that the frequency dependence has been removed (all magnitudes are evaluated at $\omega=c G / 2$ ) and that the temperature $T$ dependence has been explicitly incorporated. The latter stems from the thermal dependence of the plasma frequency and collision rate through

$$
\epsilon(T) \equiv \epsilon\left(\omega=c G / 2, \omega_{p}(T), \Gamma(T)\right),
$$

with the Drude dielectric function given by Eq. (1), and through [from Eq. (12)]

$$
s_{0}(T) \equiv \frac{1-\epsilon(T)}{\delta(T) \epsilon(T)}\left\{1-[\delta(T)]^{2}\right\}^{1 / 2} .
$$

The two first Fourier terms of the series expansion of the surface profile function appearing in Eq. (13) are given, in the case of rectangular grooves, by

$$
f_{0}=\frac{2 h A}{d}, \quad f_{1}=\frac{h}{\pi} \sin \left(2 \pi \frac{A}{d}\right) .
$$

Incidentally, recall that Eq. (13) reduces to the value expected from the SPP dispersion relation (3) on a planar surface $\left(s_{0}=0\right)$, in the limit that $|\epsilon| \gg 1$ [as imposed by the impedance boundary condition (5)].

The position of the gap is of course governed by the grating period (Bragg scattering). From now on, we choose a fixed period of $d=442 \mu \mathrm{m}$, which coincides with that of the experimental results of Refs. 5 and 6, and yields a SPP gap at about $0.3 \mathrm{THz}$. In addition, all calculations are done for intrinsic indium antimonide ( $\mathrm{InSb}$ ), due to the ease with which its plasma frequency and carrier mobility can be modified (see Table I). Indium antimonide is a semiconductor with high mobility electrons and with a small band gap. Even at room temperature there is a significant number of thermally excited carriers $\left(\simeq 10^{16}\right)$, conferring to InSb a metallic character at terahertz frequencies. In Fig. 2, we show the temperature variation (about room temperature) of the gap, given by Eq. (13) [but expressed in terms of the normal frequency $\nu=\omega /(2 \pi)$, as done for all frequencies throughout the rest of paper], for rectangular grooves of depth $h=60 \mu \mathrm{m}$ and vari-
TABLE I. Plasma frequency $\left(\omega_{p}\right)$ and collision rate $(\Gamma)$ of $\mathrm{InSb}$ as defined in Drude's formula (1) for different temperatures (Ref. 28). The high-frequency term of the permittivity is $\epsilon_{\infty}=15.75$.

\begin{tabular}{lcc}
\hline \hline Temperature $(\mathrm{K})$ & $\omega_{p} /(2 \pi)(\mathrm{THz})$ & $\Gamma /(2 \pi)(\mathrm{THz})$ \\
\hline 225 & 3.1 & 0.15 \\
240 & 4.0 & 0.18 \\
255 & 5.1 & 0.21 \\
270 & 5.9 & 0.23 \\
280 & 6.7 & 0.24 \\
295 & 8.0 & 0.26 \\
310 & 9.0 & 0.28 \\
325 & 9.9 & 0.31 \\
\hline \hline
\end{tabular}

ous widths. The most significant effect is the gap broadening with decreasing temperature. In essence, upon reducing the temperature, the dielectric function of InSb becomes less negative (smaller $|\epsilon|$, due to the decrease in the free carrier density, leading to a lower plasma frequency; see Table I); thus, the skin depth $\delta$ increases with decreasing temperature, which in turn results in larger scattering cross sections for the same grooves (the SPP feels the groove more intensely). Furthermore, the gap broadening is strongly asymmetric: $\nu_{+}$ barely varies with decreasing temperature, whereas $\nu_{-}$diminishes. A similar effect (with increasing groove sizes) has been explained by Barnes et al. in Ref. 8 and reported elsewhere. ${ }^{11,12,16}$ (Recall also that there is another temperature effect on the gap due to the decrease of the (flat surface) SPP frequency [cf. Eq. (3a)] at $k=G / 2$ induced by the change in the permittivity with decreasing temperature, however minor compared with the latter grating-induced gap opening.) Therefore, it is evident from Fig. 2 that the shift of the lower gap edge can be in principle exploited to achieve SPP thermal switching.

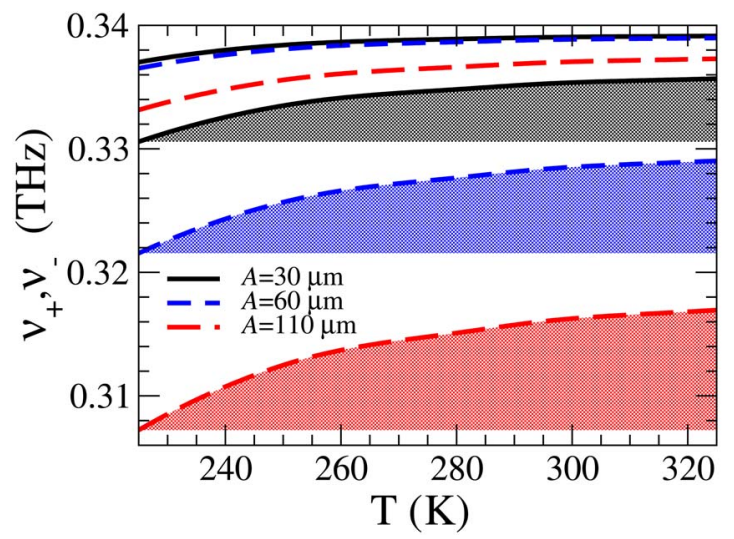

FIG. 2. (Color online) Thermal dependence of the first gap of SPP on InSb arrays (period $d=442 \mu \mathrm{m}$ ) of rectangular grooves. The lines represent the lower $\nu_{-}$and higher $\nu_{+}$band edges. The grooves have a depth of $h=60 \mu \mathrm{m}$ and half-width: $A=30 \mu \mathrm{m}$ (solid curves), $A=60 \mu \mathrm{m}$ (dotted curves), and $A=90 \mu \mathrm{m}$ (dashed curves). Shaded areas indicate the spectral region where the thermally-induced shift of the lower band edge occurs. 


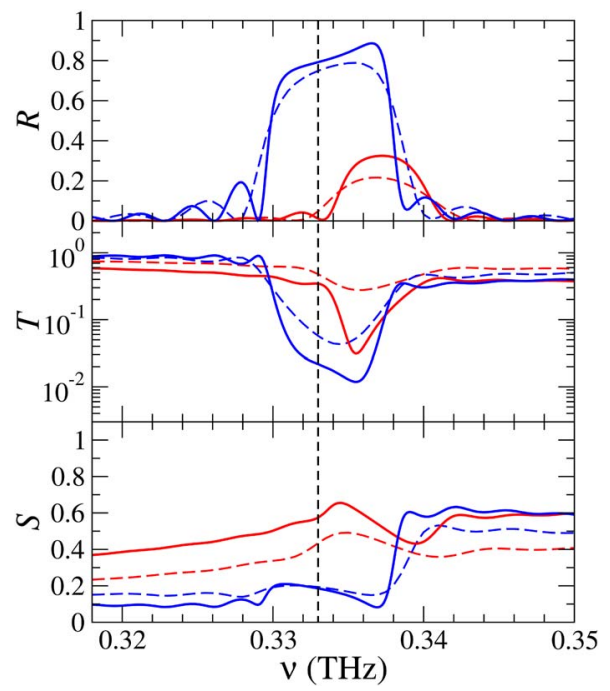

FIG. 3. (Color online) Spectral dependence of the SPP reflection $(R)$ and transmission $(\mathcal{T})$ coefficients and of the normalized radiative losses $(S)$, for arrays (period $d=442 \mu \mathrm{m}$ ) consisting of a finite number $N$ of square grooves $2 A=h=60 \mu \mathrm{m}$, for two different temperatures: $T=225 \mathrm{~K}$ (blue) and $325 \mathrm{~K}$ (red). $N=60$ (dashed curves) and $N=90$ (solid curves).

\section{SPP REFLECTION AND TRANSMISSION SWITCHING}

Let us now explore how all the SPP scattering channels behave with temperature in the case of finite arrays of $N$ grooves, thereby shedding light onto the manipulation of SPP thermal switching. To this end, we numerically calculate the SPP reflection and transmission coefficients and the radiative losses, as described above, in a variety of configurations and different temperatures. Near-field calculations are also carried out. Absorption losses are neglected in the calculations in order to yield meaningful scattering coefficients (unitarity is satisfied within $0.5 \%$ ), which is in turn fully justified by the fact that the imaginary part of the SPP wave vector [cf. Eq. (3)] is very small (the SPP inelastic length thus being much larger than the array lengths studied below). This is straightforwardly implemented in the formulation by assuming that the imaginary part of the dielectric function is negligible, as long as $|\operatorname{Re}(\epsilon)| \gg \operatorname{Im}(\epsilon)$ (Re and $\operatorname{Im}$ denote, respectively, real and imaginary parts). Nevertheless, for the actual dielectric function of InSb [cf. Eq. (1) and Table I], $|\operatorname{Re}(\epsilon)| \sim \operatorname{Im}(\epsilon)$. If we simply take $\operatorname{Im}(\epsilon) \equiv 0$ in calculating the SPP wave vector (3), it is evident that the approximate value of $k(\omega)$ thus obtained differs substantially from the true value. Therefore, it is by all means more accurate to make use of an effective real dielectric function $\epsilon_{\text {eff }}$ that exactly yields the same $\operatorname{Re}[k(\omega)]$.

\section{A. Long arrays of small grooves}

First, we represent $R(\nu), \mathcal{T}(\nu)$, and $S(\nu)$ in Fig. 3 for long but finite arrays with different numbers of square grooves $(2 A=h=60 \mu \mathrm{m})$ with the same period $d=442 \mu \mathrm{m}$, for two different temperatures $T(\mathrm{~K})=225$ and 325 . As expected from the SPP band structure, 8,12 a similar spectral variation is observed for all curves: a low-frequency region of negligible reflection, large transmission, and some amount of radiative leakage, followed by a narrow range $(\sim$ gap $)$ of low transmission (and significant reflection, up to $R \sim 90 \%$, and/or radiative leakage), beyond which the reflection becomes negligible again and both transmission and radiation increase. The latter high-frequency region corresponds to the upper SPP allowed band, which indeed enters in the radiative region, so that radiative leakage increases with array length $(L=N d)$, thus limiting SPP transmission. The behavior of $R(\nu), \mathcal{T}(\nu)$, and $S(\nu)$ in Fig. 3 for fixed temperature upon increasing the number of grooves is similar to that for visible SPP's on Ag nanodefect arrays. ${ }^{16}$ A minimum number of grooves (which in turn depends on the groove scattering cross section $\left.{ }^{16}\right)$ is required for Bragg scattering to fully build up the gap (see Fig. 3 for $N=90$ ); this is revealed by the abrupt changes of $R(\nu), \mathcal{T}(\nu)$, and $S(\nu)$ at the band edges, reaching their saturation values within the gap. Fewer grooves ( $N=60$ in Fig. 3) produce a weaker gap, with band edges smeared out. Other finite-size effects are Fabry-Perottype oscillations near the band edges.

We would like to emphasize in Fig. 3 the temperature dependence of the gap. If we focus on the curves for a fixed number of grooves-e.g., $N=60$-we observe that, at a given frequency range $\nu(\mathrm{THz}) \in[0.328,0.335]$, by heating or cooling the InSb sample, SPP switching can be achieved between reflection and transmission. In particular, at $\nu$ $=0.333 \mathrm{THz}$, the InSb groove array behaves as a SPP thermal mirror switch with large switching efficiency; i.e., it allows large transmission at high temperature $T=325 \mathrm{~K}(\mathcal{T}$ $\sim 70 \%$ and $R \sim 5 \%$ ), and upon being cooled down to $T$ $=225 \mathrm{~K}$ becomes an efficient mirror $(\mathcal{T} \sim 5 \%$ and $R=80 \%)$, resulting in large switching efficiencies in reflection and transmission.

To illustrate the physical mechanism underlying the SPP thermal switching, we show in Fig. 4 the electric field intensity in the vacuum near-field region immediately above the InSb groove array (see Fig. 1) consisting of $N=60$ square grooves as in Fig. 3, when a SPP of frequency $\nu$ $=0.333 \mathrm{THz}$ impinges from the left-hand side; the three nearfield maps (with the same color scale) correspond to different temperatures $T(\mathrm{~K})=225,270,325$. The common features to all maps with regard to the $x$ axes (parallel to the interface) dependence are (i) for $x<-N d / 2=-13.26 \mu \mathrm{m}$, an interference pattern of period $\sim \pi / k(\nu)$ arises from the incident and reflected SPP given by the inverse SPP wave vector on a plane [Eq. (3)]; (ii) for $|x|<N d / 2$, the pattern is similar, except for some mismatch at the array borders, to the SPP behavior within an infinite array [expected Bloch pattern with a rapid periodic component and a slowly varying envelope, either oscillating (band) or exponentially decaying (gap)]; (iii) for $x>N d / 2=13.26 \mu \mathrm{m}$, a constant intensity, transmitted SPP. Note also in all cases the exponential decay of the intensity away and perpendicular to the interface due to the evanescent character of the SPP, along with some propagating, radiative leakage originated from within the groove array.

Let us analyze the distinct features in Fig. 4 associated with the temperature change. For $T=325 \mathrm{~K},\left|\epsilon_{\mathrm{InSb}}\right|$ is higher, the penetration depth of the SPP into vacuum is larger, as 

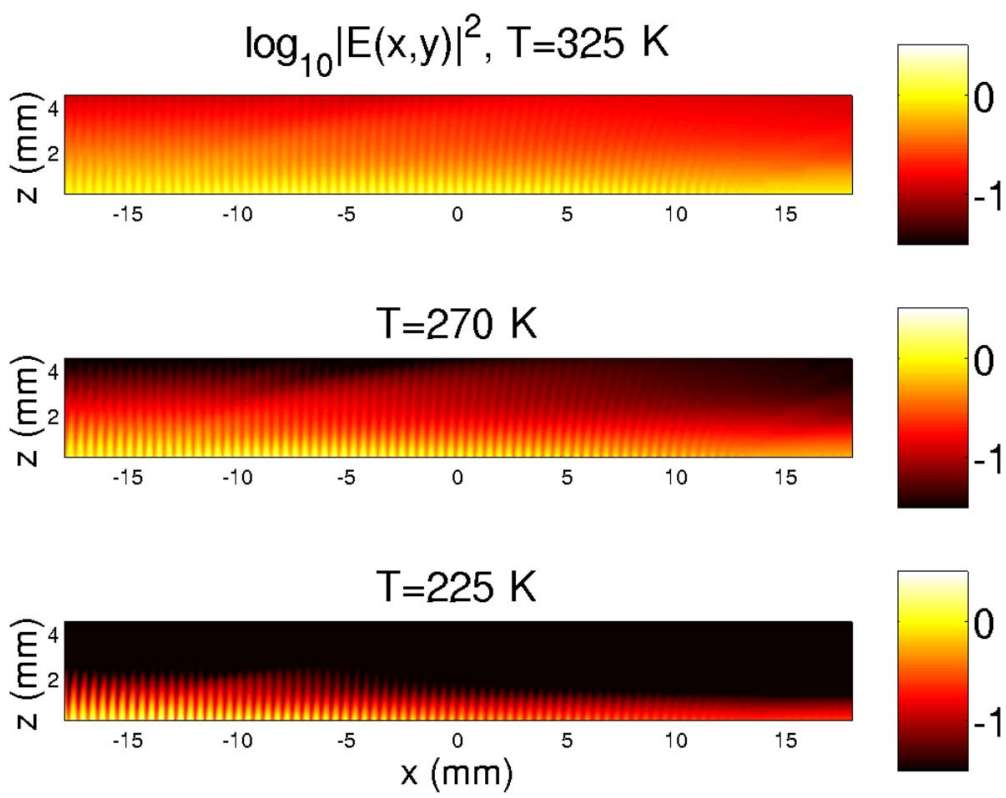

FIG. 4. (Color online) Near (electric) field intensity (log scale) images in an area of 36.4 $\times 4.5 \mu \mathrm{m}^{2}$ (in vacuum) perpendicular to the InSb surface for an array (period $d=442 \mu \mathrm{m}$ ) of $N$ $=60$ square grooves $(2 A=h=60 \mu \mathrm{m})$. A monochromatic SPP of frequency $\nu=0.333 \mathrm{THz}(\lambda$ $=901 \mu \mathrm{m})$ impinges from the negative $x$ axes onto the groove array, located at the bottom center (see Fig. 1). (a) $T=325 \mathrm{~K}$, (b) $T=270 \mathrm{~K}$, and (c) $T=225 \mathrm{~K}$. revealed in the smaller near-field decay away from the interface, and the correspondingly lower skin depth $\delta$ into InSb makes the SPP barely feel the groove array. The SPP propagate through the array since the incident SPP thus matches a propagating mode for the infinite groove array, leading to large SPP transmission, with small SPP reflection (see the low visibility of the fringes for $x<-13.26 \mu \mathrm{m}$ ) and some radiative leakage. As the temperature is decreased (see Fig. 4 for $T=270 \mathrm{~K}),\left|\epsilon_{\mathrm{InSb}}\right|$ diminishes, the penetration depth decreases, and the larger skin depth leads to larger scattering cross sections (the SPP mode for the groove array approaches a band edge), so that the SPP is scattered into reflected SPP and radiative leakage at the expense of SPP transmission. If the temperature is cooled down to $T$ $=225 \mathrm{~K},\left|\epsilon_{\mathrm{InSb}}\right|$ becomes much smaller and the penetration depth into vacuum (InSb) small (large) enough, so that the SPP is strongly backscattered by the groove array $(R$ $\sim 90 \%$ ) due to the SPP gap.

\section{B. Short arrays of larger grooves}

The high thermal switching efficiencies (specially relevant in reflection) for the array of subwavelength grooves, as shown above, have been achieved with a large number of grooves $N=60$, which yields an array length of $\sim 26.5 \mathrm{~mm}$. Fewer grooves would make the array less effective for switching, as revealed in Fig. 3. If a shorter device is needed, larger grooves (with larger scattering cross sections) could in principle be used. We show in Fig. 5 the SPP reflection, transmission, and radiation coefficients as in Fig. 3 but for arrays of $N=30$ and 40 rectangular grooves $(A=h=60 \mu \mathrm{m})$. The basic spectral behavior of all SPP scattering channels mentioned above is reproduced for such wider grooves, with the following exceptions. First, SPP gaps are broader, as expected from Eq. (13) and Fig. 2. Second, the number of grooves required for the SPP gap to be fully formed is significantly smaller. And third, the magnitudes of $R, \mathcal{T}$, and $S$ are of course different. Actually, radiative losses are substan- tially larger in the low-frequency region (lower SPP band) and, what is even more relevant, within the SPP gap; an analogous effect has been reported for visible SPP on Ag nanodefect arrays. ${ }^{16}$ This is the reason why SPP reflection does not exceed $R \leq 70 \%$ at best $(S \geq 30 \%)$. Still, SPP switching is of course achievable exploiting the SPP gap shift with temperature $\nu(\mathrm{THz}) \in[0.318,0.328]$ (see Fig. 2).

If an incoming SPP at $\nu=0.323$ is chosen, as shown in the near-field maps in Fig. 6, by cooling down the InSb sample, the SPP switches from large transmission (negligible reflection) to negligible transmission and significant reflection $R$ $\sim 55 \%$, as evidenced by the high-visibility fringes in the negative- $x$ axis for $T=225 \mathrm{~K}$. The same physical mechanisms as mentioned with regard to Fig. 4 govern the SPP thermal switching, but the larger scattering cross sections of

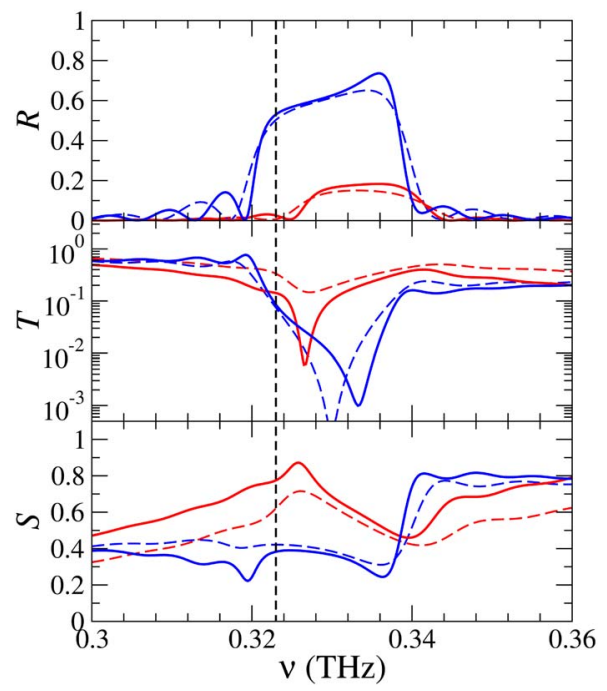

FIG. 5. (Color online) Spectral dependence of the SPP reflection $(R)$ and transmission $(\mathcal{T})$ coefficients and of the normalized radiative losses $(S)$, as in Fig. 3, but for arrays of rectangular grooves $A=h=60 \mu \mathrm{m}: N=30$ (dashed curves) and $N=40$ (solid curves). 

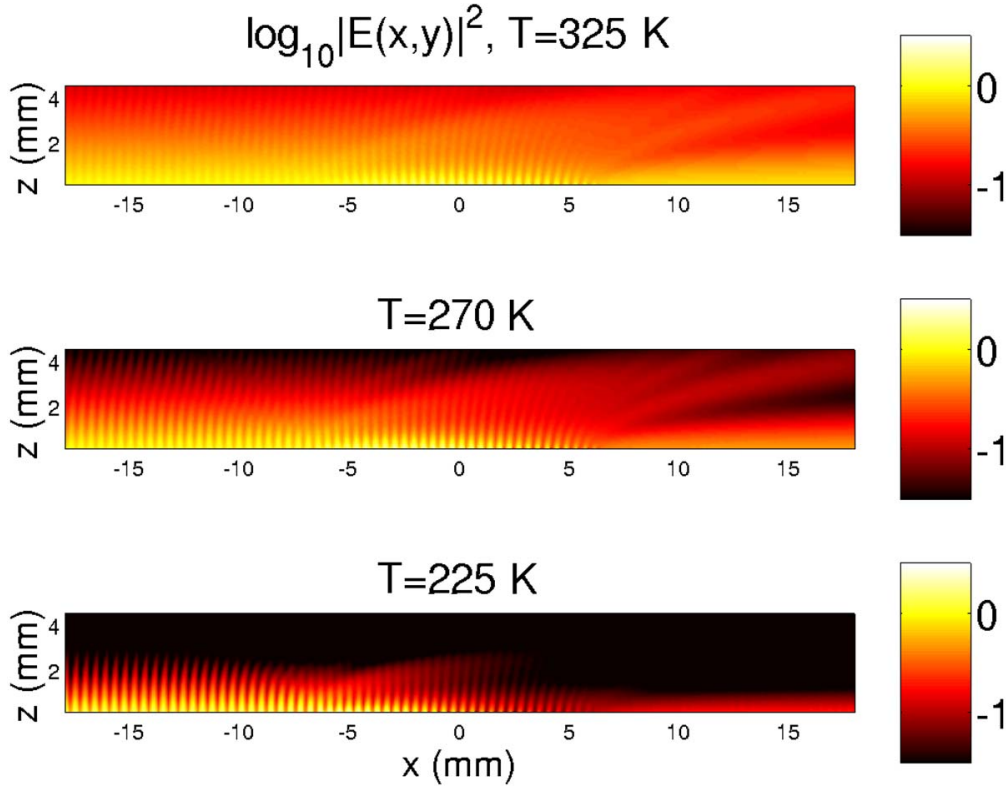

FIG. 6. (Color online) Near (electric) field intensity (log scale) images as in Fig. 4, but for an array (period $d=442 \mu \mathrm{m}$ ) of $N=30$ rectangular grooves $(A=h=60 \mu \mathrm{m})$, the incident SPP frequency being $\nu=0.323 \mathrm{THz}(\lambda=929 \mu \mathrm{m})$. (a) $T$ $=325 \mathrm{~K}$, (b) $T=270 \mathrm{~K}$, and (c) $T=225 \mathrm{~K}$. individual grooves lead to larger losses in the form of radiative leakage. This radiation leakage is apparent in Fig. 4 by the near-field intensities within the groove array, $|x|$ $<6.63 \mathrm{~mm}$, even for the lower temperature. Nonetheless, recall that only $N=30$ grooves (array length $\sim 13.26 \mathrm{~mm}$ ) are needed to form the gap, which might be advantageous in experimental configurations where shorter devices are required and/or absorptive losses are significant.

Wider, deeper grooves could be employed if shorter arrays are needed, but at the expense of increasing radiative losses. Grooves narrower and/or shallower than those used in Fig. 3 will give rise to even neater SPP thermal switchinghowever, requiring very long arrays for which absorption might become significant. With regard to groove size and shapes, we have verified that, as long as groove dimensions are subwavelength, as required both for exploiting the first SPP gap and also for the impedance boundary condition we are using to correlate with the groove profile, SPP scattering basically depends on the profile cross section, leading to similar efficiencies for, e.g., rectangular, top-hat, and Gaussian grooves and ridges with identical cross sections (not shown here).

Concerning radiative losses, we have calculated the angular distribution of intensity scattered into vacuum for the two groove arrays and two of the temperatures employed in Figs. 4 and 6. The corresponding DRC's are shown in Fig. 7. Basically, most of the SPP coupling into propagating waves in vacuum is concentrated at grazing angles, both positive (larger contribution, stemming from the incoming SPP) and negative (from the reflected SPP), as expected from simple first-order scattering arguments based on the parallel momentum transferred. Angular oscillations arise that are due to the Fabry-Perot-like interference within the finite arrays, with period inversely proportional to the array length and thus smaller for the larger arrays. It is also evident that the shorter array (recall that they consist of larger grooves) produce higher radiative losses.

\section{Half-period grooves}

Finally, we present some results obtained for an array $(d$ $=442 \mu \mathrm{m})$ of $N=30$ rectangular grooves with width $2 A$ $=220 \mu \mathrm{m} \sim d / 2$ and depth $h=60 \mu \mathrm{m}$, as used in obtaining the experimental results of Ref. 6. It should be emphasized that the groove dimensions are such that the theoretical approach employed in this work is no longer valid [since it does not suffice to retain the lowest order term of the surface impedance $^{23}$ as in Eq. (12)]. Actually, rigorous numerical calculations have been employed to reproduce the experimental results. ${ }^{6}$ However, the physical insight that the reduced Rayleigh method presented here provides might be useful to understand the scattering process, unlike the exact formulation in Ref. 6. For instance, we show in Fig. 8 the spectral dependence of the SPP reflection, not shown previously, ${ }^{6}$ at three different temperatures. Thereby, the SPP switching efficiencies can be obtained in reflection; note that $R \sim 40 \%$ for $T=225 \mathrm{~K}$ within the frequency range $\nu(\mathrm{THz})$ $\in[0.308,0.317]$ where the gap broadening occurs (see Fig. 2). Indeed, we know from calculations of SPP scattering in the visible ${ }^{16}$ that the use of the surface impedance boundary

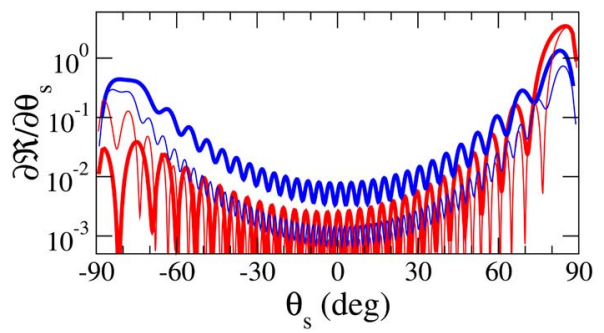

FIG. 7. (Color online) Angular distribution of scattered intensity (DRC) resulting from the SPP scattering from the groove arrays employed in Figs. 4 and 6: $T=225 \mathrm{~K}$ (blue) and $325 \mathrm{~K}$ (red). Thin curves: $N=60$ square grooves with $2 A=h=60 \mu \mathrm{m}$ at $\nu$ $=0.333 \mathrm{THz}$. Thick curves: $N=30$ rectangular grooves with $A=h$ $=60 \mu \mathrm{m}$ at $\nu=0.323 \mathrm{THz}$. 


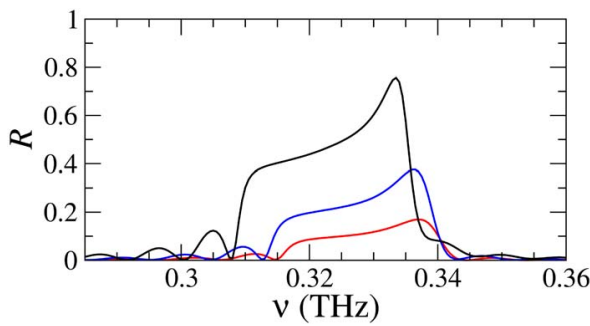

FIG. 8. (Color online) Spectral dependence of the SPP reflection $(R)$ as in Fig. 3(a) but for arrays of $N=30$ rectangular grooves of half-width $A=110 \mu \mathrm{m}$ and depth $h=60 \mu \mathrm{m}: T=225 \mathrm{~K}$ (black), $270 \mathrm{~K}$ (blue), and $325 \mathrm{~K}$ (red).

condition underestimates SPP scattering, so that the former reflection coefficients are to be taken as upper limits (radiative and ohmic losses might reduce the amount of SPP reflection within the gap).

\section{CONCLUDING REMARKS}

We have theoretically investigated the thermal variations of the THz-SPP scattering for finite arrays of sub wavelength grooves on InSb. First, a simple approximate analytical formula has been derived for the SPP gap broadening, which may help guide the choice of parameters for either experimental or numerical works. Since the thermal gap broadening is asymmetric, it is predicted that the shift of the lower band edge provides the appropriate spectral range for achieving SPP switching. A rigorous formulation is employed that stems from the reduced Rayleigh equation obtained by imposing an impedance boundary condition. ${ }^{23,24}$

This formulation straightforwardly yields all the scattering channels-namely, SPP reflection and transmission, as well as radiative leakage, energy being conserved (absorptive losses are neglected). We have carried out numerical calculations for the spectral dependence of all these channels in the vicinity of the first SPP gap for different temperatures, with the aim of determining the SPP thermal switching conditions for a variety of groove number and sizes. Our calculations show that the region of the lower SPP band edge shift is more suitable for that purpose. In addition, it has been shown that smaller grooves provide better SPP switching in both reflection and transmission due to the suppression of radiative losses. However, the smaller the groove cross section, the larger the number of grooves needed, so that a compromise between groove size and number has to be reached (recall that the array length should be well shorter than the SPP inelastic length). Larger, yet sub wavelength, grooves can of course be employed if even shorter arrays are required, with reasonable switching efficiencies, but at the expense of having the total SPP reflection reduced by increasing radiative losses. Moreover, near-field intensity maps are presented that illustrate and shed light onto the physical mechanism inducing SPP thermal switching. The resulting angular distributions of the radiative losses are also calculated. We also provide results of SPP reflection switching for exactly the same parameters used in recent experiments. ${ }^{6}$ Our formulation can be used to describe any other means of achieving SPP switching on related configurations, as long as the physical mechanism determining the change in the dielectric response of the host (or surrounding) material is properly incorporated.

Note added. After the manuscript was submitted, we learned of the paper by Søndergaard et al. ${ }^{29}$ dealing with near-IR, long-range SPP propagating through gratings consisting of gold ridges on thin films. The authors include calculations of the spectra of the SPP scattering coefficients and near-field images that qualitatively resemble some of the results presented here, although no switching mechanism is considered therein.

\section{ACKNOWLEDGMENTS}

This work was supported in part by the Spanish "Ministerio de Educación y Ciencia" (Grant Nos. BFM2003-0427 and FIS2004-0108) and "Comunidad de Madrid" (Grant MICROSERES) and by the European Union (Grant HPRN-CT2002-00206). We are grateful to M. Kuttge, P. Haring Bolivar, H. Kurz, and A. A. Maradudin for useful discussions and support. The work of J.G.R. was supported by the "Stichting voor Fundamenteel Onderzoek der Materie" (FOM), which is financially supported by the "Nederlandse Organisatie voor Wetenschappelijk Onderzoek" (NWO).
*Electronic address: j.sanchez@iem.cfmac.csic.es

${ }^{1}$ H. Raether, Surface Polaritons on Smooth and Rough Surfaces and on Gratings (Springer-Verlag, Berlin, 1988).

${ }^{2}$ W. L. Barnes, A. Dereux, and T. W. Ebbesen, Nature (London) 424, 824 (2003).

${ }^{3}$ J. Takahara and T. Kobayashi, Opt. Photonics News 15, 54 (2004).

${ }^{4}$ A. V. Zayats, I. I. Smolyaninov, and A. A. Maradudin, Phys. Rep. 408, 131 (2005).

${ }^{5}$ J. Gómez Rivas, M. Kuttge, P. Haring Bolivar, H. Kurz, and J. A. Sánchez-Gil, Phys. Rev. Lett. 93, 256804 (2004).

${ }^{6}$ J. Gómez Rivas, M. Kuttge, H. Kurz, P. Haring Bolivar, and J. A. Sánchez-Gil, Appl. Phys. Lett. 88, 082106 (2006).
${ }^{7}$ S. C. Kitson, W. L. Barnes, and J. R. Sambles, Phys. Rev. Lett. 77, 2670 (1996).

${ }^{8}$ W. L. Barnes, T. W. Preist, S. C. Kitson, and J. R. Sambles, Phys. Rev. B 54, 6227 (1996).

${ }^{9}$ F. Pincemin and J.-J. Greffet, J. Opt. Soc. Am. B 13, 1499 (1996).

${ }^{10}$ J. A. Sánchez-Gil, Phys. Rev. B 53, 10317 (1996).

${ }^{11}$ M. Kretschmann and A. A. Maradudin, Phys. Rev. B 66, 245408 (2002).

${ }^{12}$ M. Kretschman, T. A. Leskova, and A. A. Maradudin, Opt. Commun. 215, 205 (2004).

${ }^{13}$ H. Ditlbacher, J. R. Krenn, G. Schider, A. Leitner, and F. R. Aussenegg, Appl. Phys. Lett. 81, 1762 (2002). 
${ }^{14}$ H. Ditlbacher, J. R. Krenn, A. Hohenau, A. Leitner, and F. R. Aussenegg, Appl. Phys. Lett. 83, 3655 (2003).

${ }^{15}$ J. C. Weeber, Y. Lacroute, A. Dereux, E. Devaux, T. Ebbesen, C. Girard, M. U. González, and A.-L. Baudrion, Phys. Rev. B 70, 235406 (2004).

${ }^{16}$ J. A. Sánchez-Gil and A. A. Maradudin, Appl. Phys. Lett. 86, 251106 (2005).

${ }^{17}$ F. López-Tejeira, F. J. García-Vidal, and L. Martín-Moreno, Phys. Rev. B 72, 161405(R) (2005).

${ }^{18}$ S. I. Bozhevolnyi, J. Erland, K. Leosson, P. M. W. Skovgaard, and J. M. Hvam, Phys. Rev. Lett. 86, 3008 (2001).

${ }^{19}$ Y.-H. Liau, S. Egusa, and N. F. Scherer, Opt. Lett. 27, 857 (2002).

${ }^{20}$ A. V. Krasavin, K. F. MacDonald, N. I. Zheludev, and A. Zayats, Appl. Phys. Lett. 85, 3369 (2004).

${ }^{21}$ T. Nikolajsen, K. Leosson, and S. I. Bozhevolnyi, Appl. Phys.
Lett. 85, 5833 (2004).

${ }^{22}$ A. V. Krasavin and N. I. Zheludev, Appl. Phys. Lett. 84, 1416 (2004).

${ }^{23}$ A. A. Maradudin, in Topics in Condensed Matter Physics, edited by M. P. Das (Nova, New York, 1994).

${ }^{24}$ J. A. Sánchez-Gil and A. A. Maradudin, Phys. Rev. B 60, 8359 (1999).

${ }^{25}$ J. A. Sánchez-Gil, Appl. Phys. Lett. 73, 3509 (1998).

${ }^{26}$ J. A. Sánchez-Gil and A. A. Maradudin, Opt. Express 12, 883 (2004); Opt. Lett. 28, 2255 (2003).

${ }^{27}$ D. L. Mills, Phys. Rev. B 15, 3097 (1977).

${ }^{28}$ J. Gómez Rivas, C. Janke, P. Haring Bolivar, and H. Kurz, Opt. Express 13, 847 (2005).

${ }^{29}$ T. Søndergaard, S. I. Bozhevolnyi, and A. Boltasseva, Phys. Rev. B 73, 045320 (2006). 